\title{
Composite Alignment Media for the Measurement of Independent Sets of NMR Residual Dipolar Couplings
}

\author{
Ke Ruan and Joel R. Tolman* \\ Department of Chemistry, Johns Hopkins University, 3400 North Charles Street, Baltimore, Maryland 21218
}

Received August 12, 2005; E-mail: tolman@jhu.edu

Residual dipolar couplings (RDCs) have become one of the standard tools employed for biomolecular NMR structure determination due to their sensitivity to minute changes in the relative orientation of even spatially remote bonds. ${ }^{1}$ However, as a single measured RDC does not correspond to a unique bond orientation, RDC-based restraints are most easily introduced after an initial structural model has been derived using other NMR data. This ambiguity in the interpretation of the RDCs can be lifted using one of several more data intensive approaches. ${ }^{1}$ A particularly fruitful route for overcoming orientational ambiguity is to measure RDCs utilizing several different aligning media. ${ }^{2}$ The use of multiple alignment media allows complementary RDCs to be acquired, providing for the highly precise and accurate determination of bond orientations. ${ }^{3}$ Furthermore, it has been shown that if RDCs corresponding to five independent alignments are available, then the interpretation of the RDCs can be extended to the description of generalized order parameters and the magnitudes and asymmetries of motion, in addition to determination of mean bond orientations. $^{4}$

Although there are now a multitude of different media which can be employed for the purpose of introducing a weak degree of ordering of a biomolecule, ${ }^{5}$ it remains challenging to obtain a large number of independent RDC datasets. These difficulties primarily arise due to the inability to control whether the RDCs measured using a specific medium will provide novel information content relative to measurements made in other media, but also arise due to incompatibilities of the biomolecule with one or several different alignment media. In addition, the analysis of multi-alignment RDC data can potentially be complicated if the structure or dynamics of the biomolecule is perturbed by interactions with a specific alignment media. ${ }^{6}$ It would clearly be beneficial to develop the means for the modulation of molecular alignment using a single alignment medium. Toward this goal, we demonstrate here in an application to the protein ubiquitin that variations in molecular alignment can be introduced merely by changing the morphological characteristics of a composite medium composed of stretched polyacrylamide gels (SAG) ${ }^{7}$ and ordered bacteriophage Pf1 particles embedded within the gel. ${ }^{8}$ This approach preserves the microscopic nature of interactions between the biomolecule and the aligning medium and exploits the existence of an interference effect between two different mechanically ordered alignment media, which produces an aligning potential which differs from that of its constituent parts.

Utilizing a tube of approximately ellipsoidal cross-section $(7 \times$ $5 \mathrm{~mm}$ ), polymerization was initiated for a mixture of bis-acrylamide, acrylamide, and bacteriophage Pf1, with the tube fixed at a specific angle relative to an $11 \mathrm{~T}$ magnetic field. The cast gels were dried, rehydrated with a solution of ubiquitin in the desired buffer, and then stretched such that they could be accommodated within a 5 mm open-ended NMR tube. ${ }^{7 d}$ The basic sample configuration is illustrated in Figure 1. This protocol builds on existing gel-

15032 - J. AM. CHEM. SOC. 2005, 127, 15032-15033

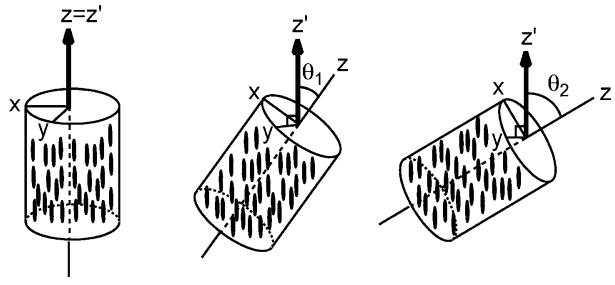

Figure 1. Variation of alignment using stretched polyacrylamide gels (SAG) and bacteriophage Pf1, which has been embedded and aligned (along $z^{\prime}$ ) at different angles relative to the long axis $(z)$ of the sample. The gels were cast in an approximately ellipsoidal (squashed cylinder) geometry, with dimensions of 5, 7, and $10 \mathrm{~mm}$ along the $x, y$, and $z$ axes, respectively. The gels were then dried, rehydrated, and stretched to fit within a $4.2 \mathrm{~mm}$ i.d. NMR tube.

Table 1. Experimental Ubiquitin Alignment Tensors Using Various Composite Pf1 and SAG Media $\left(\mathrm{pH} 6.6,25^{\circ} \mathrm{C}\right)$

\begin{tabular}{|c|c|c|c|c|c|c|c|c|c|}
\hline medium & $\mathrm{Pf} 1^{a}$ & acrylamide $^{b}$ & $\mathrm{Mg}^{2+c}$ & $\theta^{d}$ & $\begin{array}{c}A_{z z} \\
\left(\times 10^{-4}\right)\end{array}$ & $\eta$ & $\alpha$ & $\beta$ & $\gamma$ \\
\hline A & 4.0 & $5 \%$ & 10 & $55^{\circ}$ & -12.7 & 0.28 & -27.9 & 123.0 & 121.8 \\
\hline B & 4.0 & $5 \%$ & 10 & $0^{\circ}$ & -18.5 & 0.40 & -28.8 & 125.8 & 45.1 \\
\hline C & 3.0 & $5 \%$ & 10 & $30^{\circ}$ & -11.5 & 0.28 & -26.8 & 121.7 & 104.5 \\
\hline D & 3.0 & $5 \%$ & 20 & $30^{\circ}$ & -8.9 & 0.24 & -26.3 & 122.5 & 94.8 \\
\hline $\mathrm{E}$ & 3.0 & $5 \%$ & 10 & $55^{\circ}$ & -9.2 & 0.13 & -28.3 & 119.1 & 95.0 \\
\hline $\mathrm{F}$ & & $5 \%^{e}$ & & NA & 6.1 & 0.78 & -158.4 & 147.3 & -131.9 \\
\hline $\mathrm{G}^{f}$ & 3.5 & & & NA & -3.0 & 0.54 & -39.3 & 125.9 & 45.9 \\
\hline
\end{tabular}

${ }^{a}$ Concentration of bacteriophage Pf1 (mg/mL). ${ }^{b}$ Weight percentage of acrylamide employed. Gels were cast with a dimension of $5 \times 7 \times 10 \mathrm{~mm}$ unless otherwise noted. ${ }^{c} \mathrm{Mg}^{2+}$ concentration $(\mathrm{mM}) .{ }^{d}$ Angle subtended by the director of bacteriophage Pf1 alignment $\left(z^{\prime}\right)$ and the NMR tube axis $(z) .{ }^{e}$ Prestretched gel dimensions were $6 \times 6 \times 10 \mathrm{~mm} .{ }^{f}$ Data from Briggman and Tolman. ${ }^{4 \mathrm{a}}$

immobilized Pf1 media in that the gel is also stretched. Amide ${ }^{15} \mathrm{~N}-$ ${ }^{1} \mathrm{H}$ RDCs were collected for five different SAG/Pf1 preparations using the $\left[{ }^{15} \mathrm{~N},{ }^{1} \mathrm{H}\right]$-IPAP-HSQC experiment, ${ }^{9}$ followed by the determination of alignment tensors using the crystal structure coordinates of ubiquitin ${ }^{10}$ (1UBQ).

The details for the individual SAG/Pf1 media and their corresponding alignment tensors are summarized in Table 1. Clearly, the molecular alignment tensors vary according to the details of the SAG/Pf1 preparation. However, it remains possible that the observed changes in alignment tensors merely correspond to linear combinations of the SAG- and Pf1-induced alignment. Thus we carried out a singular value decomposition (SVD) analysis of the RDC data, for which the results are shown in Figure 2. To gauge the significance of the SVD results, the $Q$ value is reported for each resulting independent linear combination of the RDC data. Remarkably, the five SAG/Pf1-induced RDC datasets collected represent three independent, high-precision datasets. If RDC datasets acquired using SAG and Pf1 media alone are included, then a fourth independent dataset is present, albeit with lower precision $(Q=$ 0.52 ).

It is straightforward to reconstitute the experimental RDC datasets in terms of orthogonal linear combination (OLC) RDC datasets 


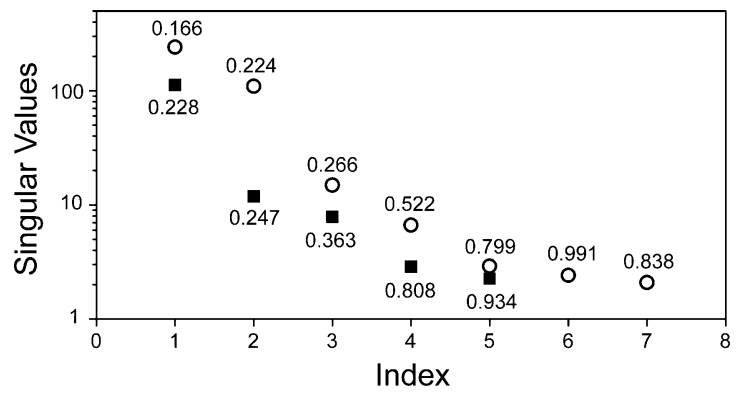

Figure 2. Singular values of RDCs measured for ubiquitin using combinations of SAG and bacteriophage Pf1 media. Each singular value is annotated with the $Q$ value, indicating the agreement between the corresponding linear combination of the RDC data and those back-calculated based on the 1UBQ crystal structural coordinates. (O) Analysis for phage Pf1 and SAG alone,

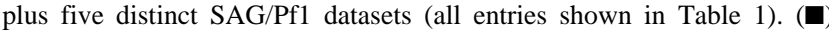
Analysis for the five distinct SAG/Pf1 datasets only (media A-E shown in Table 1).
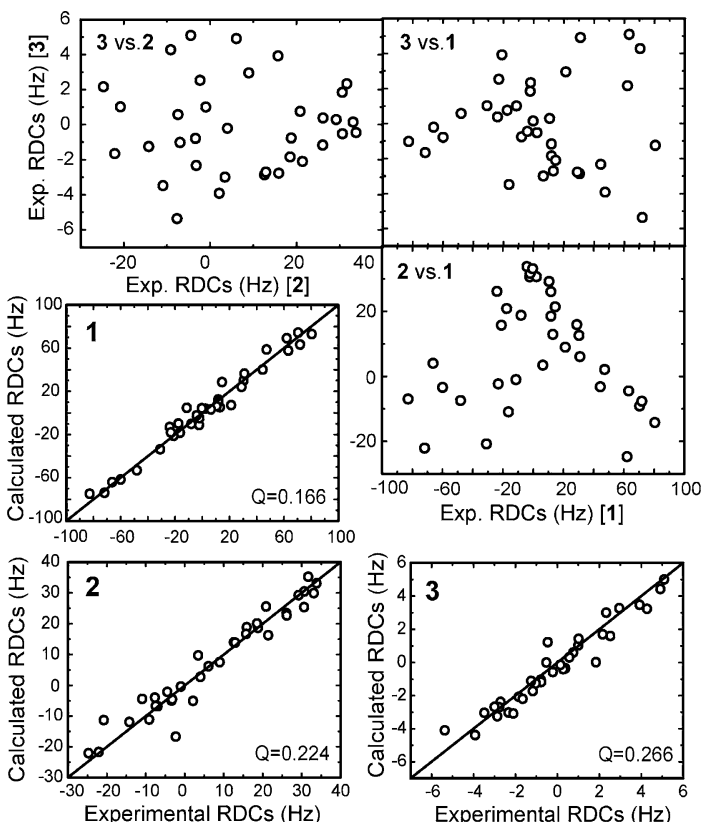

Figure 3. Illustration of the three most significant orthogonal RDC datasets resulting from a SVD analysis of all data acquired using media $A-G$ described in Table 1. These orthogonal linear combinations (OLCs) of the actually recorded RDC measurements are referred to as $\mathbf{1 , 2}$, and $\mathbf{3}$. Top right three panels: Correlation plots of each of the three linear combination datasets $(\mathbf{1}, \mathbf{2}$, and 3). Lower left three panels: Experimental versus backcalculated RDCs (using the 1UBQ X-ray coordinates) for OLC RDC datasets $\mathbf{1}, \mathbf{2}$, and $\mathbf{3}$.

based on the SVD analysis. Illustrated in Figure 3 are the three most significant OLC datasets, which are linear combinations of the seven datasets measured employing media $A-G$ (Table 1 and Supporting Information). As expected from the reported $Q$ values and their mutual orthogonality, the OLC datasets are not correlated and exhibit very good agreement with predictions based on the IUBQ solid-state coordinates. The amplitude of RDCs in the OLC datasets reflects their relative representation among the measured RDC datasets $A-G$. In the absence of a structural model, the determination as to how many of the OLC datasets are significant must be based on a consideration of the magnitude of the OLC RDCs relative to the estimated magnitude of the random errors.
This SVD-based approach is generally applicable to any analysis of multi-alignment RDC data, with signal averaging across datasets achieved by keeping only those OLC datasets which correspond to singular values of magnitude substantially greater than for the noise. ${ }^{4 a, b}$ For the present analysis of the seven datasets acquired in media $\mathrm{A}-\mathrm{G}$, a conservative choice would be to exclude OLC sets 4-7. However, based on its $Q$ value, the fourth OLC set does contain meaningful information, although with lower precision. Acquisition of further RDC data would improve the precision of this fourth OLC dataset.

The use of mechanically based alignment media allows an additional level of control of the macroscopic details of alignment. We have observed here that the combined use of two mechanically fixed media produces an interference effect between their respective aligning influences, enabling independent RDC datasets to be acquired by changing the macroscopic features of the composite medium. It appears that changes in Pf1 concentration, ionic strength, or the angle between ordering directors all influence the observed alignment. The use of such composite media provides a means by which multiple independent RDC datasets can be systematically acquired while maintaining a homogeneous environment. We are currently exploring whether the use of composite media represents a practical route to the problem of measuring five independent sets of RDC data.

Acknowledgment. We thank Ananya Majumdar for insightful comments on the manuscript, and start up funding provided by Johns Hopkins University.

Supporting Information Available: Table of RDC data. Table detailing the construction of the OLC RDC datasets. This material is available free of charge via the Internet at http://pubs.acs.org.

\section{References}

(1) (a) Prestegard, J. H.; Bougault, C. M.; Kishore, A. I. Chem. Rev. 2004, 104, 3519-3540. (b) Tolman, J. R.; Al-Hashimi, H. M. Ann. Rep. NMR Spectrosc. 2003, 51, 105-166. (c) Blackledge, M. Prog. Nucl. Magn. Reson. Spectrosc. 2005, 46, 23-61. (d) Bax, A.; Kontaxis, G.; Tjandra, N. Methods Enzymol. 2001, 339, 127-174.

(2) (a) Ramirez, B. E.; Bax, A. J. Am. Chem. Soc. 1998, 120, 9106-9107. (b) Al-Hashimi, H. M.; Valafar, H.; Terrell, M.; Zartler, E. R.; Eidsness, M. K.; Prestegard, J. H. J. Magn. Reson. 2000, 143, 402-406.

(3) (a) Ulmer, T. S.; Ramirez, B. E.; Delaglio, F.; Bax, A. J. Am. Chem. Soc 2003, 125, 9179-9191. (b) Clore, G. M.; Schwieters, C. D. Biochemistry 2004, 43, 10678-10691. (c) Bernado, P.; Blackledge, M. J. Am. Chem. Soc. 2004, 126, 7760-7761.

(4) (a) Briggman, K. B.; Tolman, J. R. J. Am. Chem. Soc 2003, 125, $10164-$ 10165. (b) Tolman, J. R. J. Am. Chem. Soc. 2002, 124, 12020-12030. (c) Meiler, J.; Prompers, J. J.; Peti, W.; Griesinger, C.; Bruschweiler, R. J. Am. Chem. Soc. 2001, 123, 6098-6107. (d) Meiler, J.; Peti, W.; Griesinger, C. J. Am. Chem. Soc. 2003, 125, 8072-8073. (e) Peti, W. Meiler, J.; Bruschweiler, R.; Griesinger, C. J. Am. Chem. Soc. 2002, 124 , $5822-5833$

(5) Prestegard, J. H.; Kishore, A. I. Curr. Opin. Chem. Biol. 2001, 5, 584590.

(6) (a) Hus, J.-C.; Brueschweiler, R. J. Biomol. NMR 2002, 24, 123-132. (b) Hus, J.-C.; Peti, W.; Griesinger, C.; Bruschweiler, R. J. Am. Chem. Soc. 2003, $125,5596-5597$.

(7) (a) Tycko, R.; Blanco, F. J.; Ishii, Y. J. Am. Chem. Soc. 2000, 122, 93409341. (b) Sass, H.-J.; Musco, G.; Stahl, S. J.; Wingfield, P. T.; Grzesiek, S. J. Biomol. NMR 2000, 18, 303-309. (c) Ishii, Y.; Markus, M. A.; Tycko, R. J Biomol. NMR 2001, 21, 141-151. (d) Chou, J. J.; Gaemers, S.; Howder, B.; Louis, J. M.; Bax, A. J. Biomol. NMR 2001, 21, 377382.

(8) Trempe, J. F.; Morin, F. G.; Xia, Z. C.; Marchessault, R. H.; Gehring, K. J. Biomol. NMR 2002, 22, 83-87.

(9) Ottiger, M.; Delaglio, F.; Bax, A. J. Magn. Reson. 1998, 131, 373-378.

(10) Vijaykumar, S.; Bugg, C. E.; Cook, W. J. J. Mol. Biol. 1987, 194, $531-544$

JA055520E 